BULL. AUSTRAL. MATH. SOC.

VOL. $23(1981), 121-138$.

\title{
EXTENSIONS OF THEOREMS OF \\ GAGLIARDO AND MARCUS AND MIZEL \\ TO ORLICZ SPACES
}

\author{
GRAHAME HARDY
}

\begin{abstract}
In 1958, Gagliardo showed that if $u$ is a locally integrable function on a domain $\Omega$ satisfying the cone condition, with all weak derivatives belonging to the Lebesgue space $L_{p}(\Omega)$ $(1 \leq p<\infty)$, then $u$ belongs to $L_{p}(\Omega)$ also. We extend this result to Orlicz spaces, and use it to extend a result of Marcus and Mizel on Nemitsky operators between Sobolev spaces to OrliczSobolev spaces.
\end{abstract}

\section{Introduction}

Let $\Omega$ be a domain (that is, an open and connected set) in $R_{n}$, and $g$ a function from $\Omega \times R_{m}$ into $R$. In Marcus and Mizel [8], it is shown that, under suitable assumptions, $g$ determines a mapping from $X=w_{1, q_{1}}(\Omega) \times \ldots \times w_{1, q_{m}}(\Omega)$ into $w_{1, p}(\Omega)$. This mapping associates with every $u=\left(u_{1}, \ldots, u_{m}\right) \in X$, a function $G \in W_{1, p}$ defined by $G \circ u(x)=g\left(x, u_{1}(x), \ldots, u_{m}(x)\right)$. Two cases are considered separately in $[8]$ :

(i) $p>1$ (Theorem 2.1 of [8] and its consequences); and

Received 2 September 1980. The author wishes to express his gratitude to Dr K. Hansen, Professor M. Marcus, Dr B. Thompson, Professor R. Vyborny, and Professor $K$. Widman, for many discussions on topics related to this paper. The problem discussed in $\$ 5$ was suggested by $\mathrm{Dr}$ Thompson. 
(ii) $p=1$ (Theorem 3.1 of [8] and its consequences).

In both cases, critical use is made of the following theorem, essentially contained in Gagliardo [5]. (The notations ${ }^{\prime} x_{i}$ and $A^{\prime}$ used below will be defined fully in the next section. Roughly, $A^{\prime}(\Omega)$ is the class of functions $f$ almost everywhere equal to a function $\tilde{f}$ on $\Omega$ which is absolutely continuous on almost all line segments parallel to the axes, and $\partial_{x_{i}}^{\prime} f$ is a function almost everywhere equal to $\partial \tilde{f} / \partial x_{i} \cdot \partial_{x_{i}} f$ denotes a weak derivative.)

THEOREM 1.1. Let $1 \leq p<\infty$, and suppose $\Omega$ is a bounded domain in $\mathrm{R}_{n}$ with the cone property. Then $f: \Omega \rightarrow R$ belongs to $W_{1, p}(\Omega)$ if and only if

$$
\begin{aligned}
& \text { (i) } f \in A^{\prime}(\Omega), \\
& \text { (ii) }{ }_{x_{i}^{\prime} f \in L_{p}}(\Omega), \quad i=1, \ldots, n .
\end{aligned}
$$

Moreover, if $f \in W_{1, p}(\Omega)$, then $\partial_{x_{i}^{\prime}}^{f} f=\partial_{x_{i}} f$ almost everywhere in $\Omega$, $i=1, \ldots, n$.

(Lemma 1.4 in [8] gives a slightly more general form of the above.)

We shall show how the case $1<p<\infty$ of Theorem 1.1 remains true if the Lebesgue space $L_{p}(\Omega)$ is replaced by an orlicz space $L_{p}(\Omega)$, where $P$ now denotes an $N$-function. We shall then show how this result may be used to generalise Theorem 2.1 of [8] to a class of Orlicz-Sobolev spaces containing the original Sobolev spaces.

\section{Preliminaries}

2.1. ORLICZ SPACES. We shall use the properties of $N$-functions and Orlicz spaces as given in Krasnosel'skiY and RutickiY [7]. We shall only need to consider Orlicz spaces defined on bounded domains $\Omega \subset R_{n}$. For our purposes, it is convenient to use the characterisation of orlicz spaces given below.

(i) Let $M$ be an $N$-function. Then a measurable function $u: \Omega \rightarrow R$ belongs to the Orlicz space $L_{M}(\Omega)$ if and only if there exists a constant 
$k>0$ such that $\int_{\Omega} M[k u(x)] d x<\infty$.

Throughout we shall use the Luxemburg norm, denoted here by $\|\cdot\|_{M(\Omega)}$. With this norm, Hölder's inequality takes the form

(ii) $\int_{\Omega} u v \leq 2\|u\|_{M(\Omega)}\|v\|_{\tilde{M}(\Omega)}$, where $\tilde{M}$ denotes the $N$-function complementary to $M$.

For convenience, a few other properties are given below.

(iii) If $M$ is an $N$-function and $u \in \mathrm{R}$, then

(a) $M(\alpha u) \leq \alpha M(u)$ if $0 \leq \alpha \leq 1$; and

(b) $M(\alpha u) \geq \alpha M(u)$ if $\alpha \geq 1$.

(iv) Suppose $P, Q$ and $Q^{\dagger}$ are $N$-functions, and there exist complementary $N$-functions $R$ and $\tilde{R}$ such that the inequalities

$$
\begin{aligned}
& R(u) \leq P^{-1}[Q(\alpha u)], \\
& \tilde{R}(u) \leq P^{-1}\left[Q^{\dagger}(B u)\right],
\end{aligned}
$$

are satisfied for all $u \geq u_{0}$, where $\alpha, \beta$ and $u_{0}$ are constants. Then there exists a constant $k$ such that

$$
\|z w\|_{P} \leq k\|u\|_{Q}\|w\|_{Q^{+}} \cdot
$$

If $P$ and $R$ are $N$-functions, $Q=P \circ R$ and $Q^{\dagger}=P \circ \tilde{R}$ are $N$-functions, and it is evident that $R, P, Q$ and $Q^{+}$satisfy the conditions in (iv). For use in $\$ 5$, we note that it is also possible to choose $P$ and $Q$ such that $P<Q$ and such that both $P$ and $Q$ satisfy the $\Delta_{2}$ condition. For example, we can take $P(u)=|u|^{p}, p>1$, and $Q(u)=[(1+|u|) \ln (1+|u|)-|u|]^{p}$.

2.2. THE CONE PROPERTY. (i) DEFINITION. A domain $\Omega \subset R_{n}$ is said to have the cone property if there exists a finite cone $C$ such that each point $x \in \Omega$ is the vertex of a finite cone $C_{x}$ contained in $\Omega$ and congruent to $C$. 
The following may be proved (see Adams [1], Theorem 4.8):

(ii) Let $\Omega$ be a bounded domain in $R_{n}$ having the cone property. For each $\rho>0$ there exists a finite collection $\left\{\Omega_{1}, \Omega_{2}, \ldots, \Omega_{m}\right\}$ of open subsets of $\Omega$ such that $\Omega=\bigcup_{j=1}^{m} \Omega_{j}$, and such that to each $\Omega_{j}$ there corresponds a subset $A_{j}$ of $\bar{\Omega}_{j}$ having diameter not exceeding $\rho$, and an open parallelepiped $P_{j}$ with one vertex at 0 , such that $\Omega_{j}=\bigcup_{x \in A_{j}}\left(x+P_{j}\right)$.

The parallelepipeds $P_{j}$ are determined by $C$, and not by $\rho$.

2.3. THE CLASS $A(\Omega)$. Let $\Omega$ be a domain in $\mathrm{R}_{n} \cdot A(\Omega)$ denotes the class of real measurable functions on $\Omega$ such that, for almost every line $\tau$ parailel to any coordinate axis, $u$ is locally absolutely continuous on $\tau \cap \Omega$ (that is, $u$ is absolutely continuous on each compact subinterval of $\tau \cap \Omega) . A^{\prime}(\Omega)$ denotes the class of functions $u$ such that $u$ coincides almost everywhere in $\Omega$ with a function $\tilde{u}$ in $A(\Omega)$. For $u \in A^{\prime}(\Omega), \partial_{x_{i}^{\prime}}^{\prime} u$, the strong approximate derivative of $u$ with respect to $x_{i}$, denotes any member of the equivalence class of functions measurable on $\Omega$ which contains $\partial \tilde{u} / \partial x_{i}$.

2.4. ORLICZ-SOBOLEV SPACES. (i) We shall use the notation $\partial_{x_{i}} u(x)$ to denote the $i$ th distribution derivative of $u: \Omega \rightarrow R$, for $\Omega \subset \mathrm{R}_{n}$. If $M$ is an $N$-function, $W^{1} L_{M}(\Omega)$ and $W^{I} E_{M}(\Omega)$ denote the classes of functions $u$ for which $u$ and $\partial_{x_{i}} u \in L_{M}(\Omega)$ and $E_{M}(\Omega)$ respectively.

(ii) We shall use the norm

$$
\|u\|_{W_{M}^{l}(\Omega)}=\|u\|_{M(\Omega)}+\sum_{i=1}^{n}\left\|\partial_{x_{i}} u\right\|_{M(\Omega)} .
$$

The following density theorem holds (see [3], Theorem 2.2). 
(iii) If $\Omega$ is a bounded domain in $R_{n}$, then $C^{\infty}(\Omega)$ is dense in $W^{I} E_{M}(\Omega)$

\section{Extension of Gagliardo's Theorem}

The statement of the theorem of Gagliardo (contained in Theorem 1.1), still holds true if the Lebesgue space $L_{p}(\Omega)$ is replaced by an Orlicz space $L_{P}(\Omega)$.

THEOREM 3.1. Let $\Omega$ be a bounded domain in $\mathrm{R}_{n}$ with the cone property, and let $P$ be an $N$-function. Then if $u \in A^{\prime}(\Omega)$ and $\partial_{x_{i}}^{\prime} u \in L_{P}(\Omega), u \in L_{P}(\Omega)$ also.

The proof follows from the sequence of lemmas below.

LEMMA 3.2. Let $\Phi$ be a one-to-one tronsformation of a domain $\Omega$ in $\mathrm{R}_{n}$ onto a domain $G$ in $\mathrm{R}_{n}$, having inverse $\Psi$. Suppose $\Phi$ and $\Psi$ have continuous derivatives on $\bar{\Omega}$ and $\bar{G}$ respectively, and let

$$
0<c=\min \left\{1, \inf _{x \in \Omega}\left|\operatorname{det} \Phi^{\prime}(x)\right|\right\}, c=\max \left\{1, \sup _{x \in \Omega}\left|\operatorname{det} \Phi^{\prime}(x)\right|\right\} .
$$

Suppose $u: \Omega \rightarrow R$ is measurable, and that the function $A u: G \rightarrow R$ is defined by

$$
A u(y)=u(\Psi(y)) .
$$

Then if $P$ is an $N$-function,

$$
c\|u\|_{P(\Omega)} \leq\|A u\|_{P(G)} \leq c\|u\|_{P(\Omega)} .
$$

Proof. For $\lambda>0,2.1$ (iii) (a) gives

$$
\begin{aligned}
\int_{\Omega} P[u(x) / \lambda] d x \leq \int_{\Omega} c P[u(x) / c \lambda] d x & \leq \int_{\Omega} P[u(x) / c \lambda]\left|\operatorname{det} \Phi^{\prime}(x)\right| d x \\
& =\int_{G} P[A u(y) / c \lambda] d y ;
\end{aligned}
$$

whence, from 2.1 (iii) (a),

$$
\|A u / c\|_{P(G)} \leq\|u\|_{P(\Omega)},
$$

which gives the first inequality. 
A similar proof, using 2.1 (iii) (b), gives the second inequality.

LEMMA 3.3. Let $\Phi$ be a non-singular linear transformation of a domain $\Omega \subset \mathrm{R}_{n}$ onto a domain $G \subset \mathrm{R}_{n}$. Then if $u$ has weak derivatives $\partial_{x_{i}} u(x), i=1, \ldots, n$, for $x \in \Omega, u \circ \Phi^{-1}$ has weak derivatives $\partial_{y_{i}}\left[u\left(\Phi^{-1}(y)\right)\right], \quad 1 \leq i \leq n$, for $y \in G$.

More general versions of the above are well-known; see, for example, Gilbarg and Trudinger ([6], page 144), or Mihailov ([9], page 124, para. 5).

Lemma 3.3 can also be easily proved directly, using the definition of a weak derivative and the change of variable formula for integrals.

LEMMA 3.4. Suppose $\Omega$ is a bounded domain in $\mathrm{R}_{n}$ having the cone property. Let $\Phi$ and $G$ be as in Lemma 3.3. Then if $u \in A^{\prime}(\Omega)$ and $\partial_{x_{i}^{\prime}}^{\prime} u \in L_{1}(\Omega), \quad l \leq i \leq n, u \circ \Psi \in A^{\prime}(G)$.

Proof. The lemma is an immediate consequence of Lemma 3.3 , and the $p=1$ case of Theorem 1.1 .

We shall use the notation $C_{\eta}(c)$ to denote a cube in $R_{n}$ with side of length $l$, having centre at $c$. If $c$ is the point $(\tau / 2, \tau / 2, \ldots, \tau / 2)$, so that one vertex is at the origin, we shall denote $c_{\eta}(c)$ by $C_{\eta}$.

LEMMA 3.5. Let $\Omega$ be a bounded domain in $\mathrm{R}_{n}$ having the cone property. Then

$$
\Omega=\bigcup_{j=1}^{m} \Omega_{j}
$$

where each $\Omega_{j}$ is an open subset of $R_{n}$ having the property (*) stated below:

(*) there exists a non-singular linear transformation $T_{j}$ such that 


$$
T_{j}\left(\Omega_{j}\right)=\underset{c \in B_{j}}{U} C_{l}(c)
$$

where $\operatorname{diam} B_{j}<1 / 8$.

Proof. Let $P_{j}, 1 \leq j \leq k$, be the parallelepipeds which occur in 2.2 (ii). Let $T_{j}, 1 \leq j \leq k$, be linear transformations which map $P_{j}$ onto $C_{1}$, and let $\pi_{j}=T_{j}^{-1}\left(C_{1 / 8 \sqrt{n}}\right)$. Let $d_{j}$ be the minimum distance between opposite faces of $\pi_{j}$, and let $\rho=\min \left\{d_{1}, \ldots, d_{k}\right\}$.

By 2.2 (ii), we may write

$$
\Omega=\bigcup_{j=1}^{k} \Omega_{j},
$$

where

$$
\Omega_{j}=\bigcup_{a \in A_{j}}\left(a+P_{j}\right)
$$

and $\operatorname{diam} A_{j}<\rho$. Thus

$$
T_{j}\left(\Omega_{j}\right)=\underset{a \in T_{j}\left(A_{j}\right)}{U}\left(a+C_{1}\right) .
$$

Each $A_{j}$ may be enclosed in a translate of $\pi_{j}$, and $T_{j}\left(A_{j}\right)$ is a subset of a cube of side $1 / 8 \sqrt{n}$, so that

$$
T_{j}\left(\Omega_{j}\right)=\operatorname{U}_{c \in B_{j}} C_{1}(c)
$$

where $\operatorname{diam} B_{j}<1 / 8$.

LEMMA 3.6. Let $\Omega \subset R_{n}$, and suppose $\Omega=\bigcup_{i=1}^{m} \Omega_{i}$. Let $P$ be an $N$-function, and let $u: \Omega \rightarrow \mathrm{R}$ be measurable. Then if $\|u\|_{P\left(\Omega_{i}\right)}<\infty$, $1 \leq i \leq m,\|u\|_{P(\Omega)}<\infty$ also.

Lema 3.6 is easily proved from 2.1 (i).

Lemmas $3.4,3.5$ and 3.6 show that it is sufficient to prove Theorem 
3.1 under the assumption that $\Omega$ is of the form

$$
\Omega=\bigcup_{c \in B} C_{1}(c)
$$

where $\operatorname{diam} B<1 / 8$.

LEMMA 3.7. Suppose $\Omega$ is a domain in $\mathrm{R}_{n}$ of the form (*), that is,

$$
\Omega=\underset{c \in B}{U} C_{1}(c)
$$

where $B \subset R_{n}$, and $\operatorname{diam} B<1 / 8$. Then $\Omega$ has the property (**) below:

(**) there exists an open set $D$ of positive measure, where $D \subset \Omega$, such that if $\alpha=\left(\alpha_{1}, \ldots, \alpha_{n}\right) \in D$ and if $x=\left(x_{1}, \ldots, x_{n}\right)$ is any point of $\Omega, \alpha$ and $x$ can be joined by a path consisting of $n$ or less straight line segments $S_{1}, S_{2}, \ldots, s_{n}$, parallel to the axes, joining the points $\left(\alpha_{1}, \alpha_{2}, \ldots, \alpha_{n}\right)$ to $\left(x_{1}, \alpha_{2}, \ldots, \alpha_{n}\right)$, $\left(x_{1}, \alpha_{2}, \alpha_{3}, \ldots, \alpha_{n}\right)$ to $\left(x_{1}, x_{2}, \alpha_{3}, \ldots, \alpha_{n}\right), \ldots$, and $\left(x_{1}, \ldots, x_{n-1}, \alpha_{n}\right)$ to $\left(x_{1}, \ldots, x_{n-1}, x_{n}\right)$ respectively, where each line segment $S_{i}$ lies in $\Omega$ and has length Zess than 1 .

Proof. Let $\gamma \in B$. Since diam $B<1 / 8$, it follows that $C_{\frac{1}{2}}(\gamma) \subset \bigcap_{c \in B} C_{1}(c)$. Let $\alpha \in C_{\frac{1}{2}}(\gamma)$, and let $x \in \Omega$. Since $x$ belongs to some cube $C_{1}(\delta)$, where $\delta \in B$, and $\alpha \in C_{1}(\delta)$ also, $\alpha$ and $x$ may be joined by a path of the form required. Hence we may take $D=C_{\frac{1}{2}}(\gamma)$.

Thus we need only prove Theorem 3.1 for domains having the property $(* *)$. We do this in the final lemma.

For $\Omega \subset \mathrm{R}_{n}$, we shall use the notation $\Omega\left(x_{i}, \ldots, x_{n}\right)$ to denote the set of points $\left(x_{1}, \ldots, x_{i-1}\right)$ such that $x=\left(x_{1}, \ldots, x_{n}\right) \in \Omega$, and $\Omega_{1,2, \ldots, i}$ to denote the projection of $\Omega$ on the hyperplane $x_{1}=0, \ldots, x_{i}=0$. Note that 


$$
\left[\Omega\left(\alpha_{i+1}, \ldots, \alpha_{n}\right)\right]\left(x_{1}, \ldots, x_{i-1}\right)=\Omega\left(x_{1}, \ldots, x_{i-1}, \alpha_{i+1}, \ldots, \alpha_{n}\right) .
$$

LEMMA 3.8. Suppose $\Omega$ is a domain in $\mathrm{R}_{n}$ having the property (**) stated in Lemma 3.7. Suppose $u \in A^{\prime}(\Omega)$, and that $P$ is an N-function. Then if ${ }_{x_{i}^{\prime}}^{\prime} u \in L_{P}(\Omega), \quad 1 \leq i \leq n, u \in L_{P}(\Omega)$ also.

Proof. By 2.1 (i), for each $i, 1 \leq i \leq n$, there exists a $k_{i}>0$ such that

$$
\int_{\Omega} P\left[(n+1) k_{i} \partial_{x_{i}^{\prime}} u\right]<\infty
$$

Since $P$ is an increasing function, (i) still holds if we replace $k_{i}$ by $k=\min \left(k_{1}, \ldots, k_{n}\right)$.

Let $\tilde{u} \in A(\Omega)$ be such that $\tilde{u}=u$ almost everywhere in $\Omega$ and $\partial \tilde{u} / \partial x_{i}=\partial_{x_{i}}^{\prime} u$ almost everywhere in $\Omega$, so that

$$
\int_{\Omega} P\left[(n+1) k\left(\partial \tilde{u} / \partial x_{i}\right)\right]<\infty
$$

also. Since $\tilde{u} \in A(\Omega)$, there exists a null subset $N_{0}$ of $\Omega$ such that $\tilde{u}(\alpha)$ is finite for all $\alpha=\left(\alpha_{1}, \ldots, \alpha_{n}\right) \in \Omega-N_{0}$. Further, since $P \geq 0$, we may write (ii) in the form

$$
\begin{aligned}
& \int_{\Omega_{1}, \ldots, i} d x_{i+1}, \ldots, d x_{n} \int_{\Omega\left(x_{i+1}, \ldots, x_{n}\right)} \\
& \quad \times P\left[(n+1) k\left(\partial \tilde{u} / \partial x_{i}\right)\left(x_{1}, \ldots, x_{i}, x_{i+1}, \ldots, x_{n}\right)\right] d x_{1} \ldots d x_{i}<\infty
\end{aligned}
$$

for each $i, 1 \leq i \leq n$. (iii) shows that there exists a null subset $\tilde{N}_{i}$ of each $\Omega_{1, \ldots, i}$ such that

$$
\text { (iv) } \begin{aligned}
&\left.\int_{\Omega\left(\alpha_{i+1}\right.}, \ldots, \alpha_{n}\right) \\
& \times P\left[(n+1) k\left(\partial \tilde{u} / \partial x_{i}\right)\left(x_{1}, \ldots, x_{i}, \alpha_{i+1}, \ldots, \alpha_{n}\right)\right] d x_{1} \ldots d x_{i}<\infty
\end{aligned}
$$

provided $\left(\alpha_{i+1}, \ldots, \alpha_{n}\right) \in \Omega_{1}, \ldots, i-\tilde{N}_{i}$. We may then choose a null set $N_{i} \subset \Omega$ such that (iv) holds for $\alpha \in \Omega-N_{i}$. Finally we choose a null 
set $N_{\tilde{u}}$ such that $\tilde{u}$ is locally absolutely continuous on line segments in $\Omega-N_{\tilde{u}}$ parallel to each axis. Put $N=N_{\tilde{u}} \cup\left(\bigcup_{i=0}^{n} N_{i}\right)$. Let $\alpha \in D-N$, where $D$ is as in Lemma 3.7. For any $x \in \Omega$, we may connect $\alpha$ to $x$ by straight line segments joining the points $\left(\alpha_{1}, \alpha_{2}, \ldots, \alpha_{n}\right),\left(x_{1}, \alpha_{2}, \ldots, \alpha_{n}\right), \ldots$,

$$
\left(x_{1}, \ldots, x_{n-1}, \alpha_{n}\right),\left(x_{1}, \ldots, x_{n}\right),
$$

and since $\tilde{u}$ is absolutely continuous on these line segments,

$$
\tilde{u}(x)=\tilde{u}(\alpha)+\sum_{i=1}^{n} \int_{\alpha_{i}}^{x_{i}}\left(\partial \tilde{u} / \partial x_{i}\right)\left(x_{1}, \ldots, x_{i-1}, t, \alpha_{i+1}, \ldots, \alpha_{n}\right) d t .
$$

Let $J_{i}$ denote the closed interval with end points $\alpha_{i}, x_{i}$, and let $\left|J_{i}\right|$ denote its length. From the convexity of $P$,

(v) $P[k \tilde{u}(x)] \leq 1 /(n+1) P[(n+1) k \tilde{u}(\alpha)]+1 /(n+1)$

$$
\times \sum_{i=1}^{n} P\left\{\int_{J_{i}}\left[(n+1) k\left(\partial \tilde{u} / \partial x_{i}\right)\left(x_{1}, \ldots, x_{i-1}, t, \alpha_{i+1}, \ldots, \alpha_{n}\right)\right] d t\right\} \text {. }
$$

For $\alpha_{i} \neq x_{i}$, Jensen's inequality shows that

$$
\begin{aligned}
P\left\{\iint_{J_{i}}\right. & {\left.\left.\left[\left|J_{i}\right|(n+1) k\left(\partial \tilde{u} / \partial x_{i}\right)\left(x_{1}, \ldots, x_{i-1}, t, \alpha_{i+1}, \ldots, \alpha_{n}\right)\right] d t\right) /\left|J_{i}\right|\right\} } \\
& \leq 1 /\left|J_{i}\right| \int_{J_{i}} P\left[\left|J_{i}\right|(n+1) k\left(\partial \tilde{u} / \partial x_{i}\right)\left(x_{1}, \ldots, x_{i-1}, t, \alpha_{i+1}, \ldots, \alpha_{n}\right)\right] d t \\
& \leq \int_{J_{i}} P\left[(n+1) k\left(\partial \tilde{u} / \partial x_{i}\right)\left(x_{1}, \ldots, x_{i-1}, t, \alpha_{i+1}, \ldots, \alpha_{n}\right)\right] d t
\end{aligned}
$$

on using 2.1 (iii) (a). Since the case $\alpha_{i}=x_{i}$ is trivial, we have 
Extensions of theorems to Orlicz spaces

131

(vi )

$$
\begin{aligned}
& P\left\{\int_{J_{i}}\left[(n+1) k\left(\partial \tilde{u} / \partial x_{i}\right)\left(x_{1}, \ldots, x_{i-1}, t, \alpha_{i+1}, \ldots, \alpha_{n}\right)\right] d t\right\} \\
& \leq \int_{J_{i}} P\left[(n+1) k\left(\partial \tilde{u} / \partial x_{i}\right)\left(x_{1}, \ldots, x_{i-1}, t, \alpha_{i+1}, \ldots, \alpha_{n}\right)\right] d t \\
& \leq \int_{\Omega\left(x_{1}, \ldots, x_{i-1}, \alpha_{i+1}, \ldots, \alpha_{n}\right)} \quad \times \quad P\left[(n+1) k\left(\partial \tilde{u} / \partial x_{i}\right)\left(x_{1}, \ldots, x_{i-1}, t, \alpha_{i+1}, \ldots, \alpha_{n}\right)\right] d t .
\end{aligned}
$$

Substituting ( $v i)$ and $(v)$ and then integrating over $\Omega$, we obtain

(vii) $\int_{\Omega} P[k u(x)] d x \leq[1 /(n+1)] P[(n+1) k \tilde{u}(\alpha)]|\Omega|+[1 /(n+1)] \sum_{i=1}^{n}$

$$
\begin{aligned}
& \times \int_{\Omega_{i}, \ldots, n} d x_{1}, \ldots, d x_{i-1} \int_{\Omega\left(x_{1}, \ldots, x_{i-1}\right)} d x_{i}, \ldots, d x_{n} \\
& \times \int_{\Omega\left(x_{1}, \ldots, x_{i-1}, \alpha_{i+1}, \ldots, \alpha_{n}\right)} \\
& \times \quad P\left[(n+1) k\left(\partial \tilde{u} / \partial x_{i}\right)\left(x_{1}, \ldots, x_{i-1}, t, \alpha_{i+1}, \ldots, \alpha_{n}\right)\right] d t .
\end{aligned}
$$

The first term on the right-hand side of (vii) is less than $\infty$, because $\Omega$ is bounded. Moreover, there exists $K_{i}<\infty$ such that

$$
\int_{\Omega\left(x_{1}, \ldots, x_{i-1}\right)} d x_{i}, \ldots, d x_{n} \leq K_{i} \text { for any }\left(x_{1}, \ldots, x_{i-1}\right) \in \Omega_{i, \ldots, n} \text {, }
$$

again because $\Omega$ is bounded. Now consider the $i$ th term, $T_{i}$ say, inside the summation sign in (vii). We may write

$$
\begin{array}{r}
T_{i}=\int_{\Omega_{i, \ldots, n}} d x_{1}, \ldots, d x_{i-1} \int_{\left[\Omega\left(\alpha_{i+1}, \ldots, \alpha_{n}\right)\right]\left(x_{1}, \ldots, x_{i-1}\right)} \\
\quad \times P\left[(n+1)\left(\partial \tilde{u} / \partial x_{i}\right)\left(x_{1}, \ldots, x_{i-1}, t, \alpha_{i+1}, \ldots, \alpha_{n}\right)\right] d t \\
\quad \times \int_{\Omega\left(x_{1}, \ldots, x_{i-1}\right)} d x_{i}, \ldots, d x_{n} \\
\leq K_{i} \int_{\Omega_{i}, \ldots, n} d x_{1}, \ldots, d x_{i-1} \int_{\left[\Omega\left(\alpha_{i+1}, \ldots, \alpha_{n}\right)\right]\left(x_{1}, \ldots, x_{i-1}\right)} \\
\quad \times P\left[(n+1) k\left(\partial \tilde{u} / \partial x_{i}\right)\left(x_{1}, \ldots, x_{i-1}, t, \alpha_{i+1}, \ldots, \alpha_{n}\right)\right] d t
\end{array}
$$

https://doi.org/10.1017/S0004972700006948 Published online by Cambridge University Press 


$$
\begin{aligned}
=K_{i} \int_{\left[\Omega\left(\alpha_{i+1}, \ldots, \alpha_{n}\right)\right]_{i, \ldots, n}} d x_{1}, \ldots, d x_{i-1} & . \\
& \times \int_{\left[\Omega\left(\alpha_{i+1}, \ldots, \alpha_{n}\right)\right]\left(x_{1}, \ldots, x_{i-1}\right)} \\
& \times P\left[(n+1) k\left(\partial \tilde{u} / \partial x_{i}\right)\left(x_{1}, \ldots, x_{i-1}, t, \alpha_{i+1}, \ldots, \alpha_{n}\right)\right] d t,
\end{aligned}
$$

where we have used the fact that $\left[\Omega\left(\alpha_{i+1}, \ldots, \alpha_{n}\right)\right]\left(x_{1}, \ldots, x_{i-1}\right)=\emptyset$ if $\left(x_{1}, \ldots, x_{i-1}\right) \in \Omega_{i, \ldots, n}-\left[\Omega\left(\alpha_{i+1}, \ldots, n\right)\right]_{i, \ldots, n}$. From (iv), $T_{i}<\infty, 1 \leq i \leq n$, so that we have shown that there exists $k>0$ such that $\int_{\Omega} P[k u(x)] d x<\infty$, whence $\|u\|_{P(\Omega)}<\infty$, by 2.1 (i).

\section{Two theorems on Orlicz-Sobolev spaces}

The following two theorems will be needed in $\S 5$. For the corresponding results in Sobolev spaces, see Lemmas 1.5 and 1.6 in Marcus and Mizel [8]. We shall use the following notation.

(i) If $\Omega$ is a domain in $\mathrm{R}_{n}, \Omega_{v}$ denotes the translate of $\Omega$ by the vector $v \in \mathrm{R}_{n}$; and for $\Omega^{\prime} \subset \mathrm{R}_{n}, \Omega^{\prime} \propto \Omega$ means that $\bar{\Omega}^{\prime}$ is a compact subset of $\Omega$. $\partial \Omega$ denotes the boundary of $\Omega$.

(ii) For $h>0, e_{i}, 1 \leq i \leq n$, the standard basis for $R_{n}$, and $x \in R_{n}$,

$$
\delta_{h}^{i} u(x)=\left(u\left(x+h e_{i}\right)-u(x)\right) / h .
$$

THEOREM 4.1. Suppose that $\Omega$ is a bounded domain in $\mathrm{R}_{n}$, that $\Omega$ ' is an open set such that $\Omega^{\prime} \propto \Omega$, and that $P$ is an $N$-function. Then if $0<h<\operatorname{dist}\left(\Omega^{\prime}, \partial \Omega\right)$, and if $u \in W^{I} E_{P}(\Omega)$,

$$
\left\|\delta_{h^{i} u}\right\|_{P\left(\Omega^{\prime}\right)} \leq\left\|\partial_{x_{i}} u\right\|_{P(\Omega)}
$$

Proof. For $u \in C^{\perp}(\Omega)$, 


$$
\left|\delta_{h}^{i} u(x)\right| \leq \int_{0}^{1}\left|\left(\partial / \partial x_{i}\right) u\left(x+h e_{i} t\right)\right|
$$

and so for $\lambda>0$, an application of Jensen's inequality gives

$$
\begin{aligned}
\int_{\Omega^{\prime}} P\left(\delta_{h^{i}} u(x) / \lambda\right) d x & \leq \int_{\Omega^{\prime}} \int_{0}^{1} P\left(\left(\partial / \partial x_{i}\right) u\left(x+h e_{i} t\right) / \lambda\right) d t d x \\
& =\int_{0}^{1} d t \int_{\Omega^{\prime}} P\left(\left(\partial / \partial x_{i}\right) u\left(x+h e_{i} t\right) / \lambda\right) d x \\
& =\int_{0}^{1} d t \int_{\Omega_{-}^{\prime} h e_{i} t} P\left(\left(\partial / \partial x_{i}\right) u(x) / \lambda\right) d x \\
& \leq \int_{0}^{1} d t \int_{\Omega} P\left(\left(\partial / \partial x_{i}\right) u(x) / \lambda\right) d x \\
& =\int_{\Omega} P\left(\left(\partial / \partial x_{i}\right) u(x) / \lambda\right) d x .
\end{aligned}
$$

Taking the infinium of all $\lambda>0$ such that the right-hand side is less than or equal to $I$ gives

$$
\left\|\delta_{h^{i}}^{i}\right\|_{P\left(\Omega^{\prime}\right)} \leq\left\|\partial u / \partial x_{i}\right\|_{P(\Omega)} .
$$

By 2.4 (iii) for any $u \in W^{l} \cdot E_{p}(\Omega)$, there exists a sequence $u_{n}$ of $C^{\infty}(\Omega)$ functions such that $u_{n} \rightarrow u$ in $W^{l} L_{p}(\Omega)$. Replacing $u$ by $u_{n}$ in the last inequality and letting $n \rightarrow \infty$ gives the result.

THEOREM 4.2. Suppose that $\Omega$ is a domain in $\mathrm{R}_{n}$, and $u \in L_{P}(\Omega)$, where $P$ is an $N$-function. Then if there exists a number $C$ such that

$$
\left\|\delta_{h^{i} u}\right\|_{P\left(\Omega^{\prime}\right)} \leq C
$$

for every open $\Omega^{\prime} \propto \Omega$ and $|h|$ sufficiently small, $\partial_{x_{i}} u \in L_{P}(\Omega)$ and $\left\|\partial_{x_{i}} u\right\|_{P(\Omega)} \leq c$

We omit the proof, as it is almost identical to that for the Lebesgue $L_{p}$ spaces, as given in, say, Agmon [2] and Friedmann [4]. 


\section{A theorem on Nemitsky operators}

We now have all the material necessary to extend Theorem 2.1 in Marcus and Mizel [8] from Lebesgue to Orlicz spaces. For convenience, we shall repeat some of the definitions from [8]. As before, $\Omega$ is a domain in $R_{n}$.

DEFINITIONS AND NOTATION 5.1. A function $g: \Omega \times R_{m}+R$ is said to be a generalised locally absolutely continuous Caratheodory function if

(i) there exists a null subset $N_{g}$ of $\Omega$ such that if $x \in \Omega-N_{g}$,

(a) $g(x, \cdot)$ is continuous in each variable separately in
$\mathrm{R}_{m}$,

(b) for every line $\tau$ parallel to one of the axes in $\mathrm{R}_{m},\left.g(x, \cdot)\right|_{\tau}$ is locally absolutely continuous;

(ii) for every fixed $t \in R_{m}, g(\cdot t) \in A^{\prime}(\Omega)$.

If "continuous in each variable separately" in (a) is replaced by "continuous", the above then defines a locally absolutely continuous Caratheodory function.

An operator $G$ on vector valued functions $u=\left(u_{1}, \ldots, u_{m}\right)$ measurable on $\Omega$, defined by

$$
G u(x)=g(x, u(x))=(g \circ u)(x)
$$

is called a Nemitsky operator.

Given $u=\left(u_{1}, \ldots, u_{m}\right): \Omega \rightarrow R_{m}$, and $N$-functions $Q_{1}, \ldots, Q_{m}$, we shall use the notation

$$
u \in W^{l} L_{\tilde{Q}^{(\Omega)}}
$$

to mean that $u_{i} \in W^{1} L_{Q_{i}}(\Omega), 1 \leq i \leq m$.

THEOREM 5.2. Let $\Omega$ be a bounded domain in $R_{n}$ having the cone property, and let $g$ be a generalised locally absolutely continuous 
Caratheodory function in $\Omega \times R_{m}$. Let $P, Q_{i}$ and $Q_{i}^{\dagger}, 1 \leq i \leq m$, be $N$-functions having the following properties:

(i) $P$ and $Q_{i}, I \leq i \leq m$, satisfy the $\Delta_{2}$ condition;

(ii) $P<Q_{i}, \quad I \leq i \leq m$;

(iii) there exist complementary $N$-functions $R_{i}$ and $\tilde{R}_{i}$ such that the inequalities

$$
R_{i}(u) \leq P^{-1}\left[Q_{i}\left(\alpha_{i} u\right)\right]
$$

and

$$
\tilde{R}_{i}(u) \leq P^{-1}\left[Q_{i}^{\dagger}\left(\beta_{i} u\right)\right]
$$

are satisfied for $u \geq u_{i}$, where $\alpha_{i}, \beta_{i}, u_{i}$,

$1 \leq i \leq m$, are constants.

Suppose $a, b, a_{k}, b_{k, j}$ are functions such that

I. for every fixed $t \in R_{m}$,

$\left|\partial_{x_{i}}^{\prime} g(x, t)\right| \leq a(x)+b(t)$ almost everywhere in $\Omega, i=1, \ldots, n$;

II. the inequality

$$
\left|\partial g(x, t) / \partial t_{k}\right| \leq a_{k}(x)+\sum_{j=1}^{m} b_{k, j}\left(t_{j}\right), k=1, \ldots, m,
$$

holds at every point $(x, t) \in\left(\Omega-N_{g}\right) \times \mathrm{R}_{n}$ at which the derivative exists in the classical sense.

Furthermore, $a, b, a_{k}$ and $b_{k, j}$ have the properties (iv)-(viii) Zisted below:

(iv) $0 \leq a \in L_{P}(\Omega)$;

(v) b is non-negative and separately continuous in $\mathrm{R}_{m}$;

(vi) $0 \leq a_{k} \in L_{Q_{k}^{\prime}}(\Omega), \quad 1 \leq k \leq m$; 
(vii) $0 \leq b_{k, j}$ is an extended real valued Borel function on $R, k, j=1, \ldots, m$;

(viii) $b_{k, k} \in L_{1}^{10 c}(R), k=1, \ldots, m$.

Let $u=\left(u_{1}, \ldots, u_{m}\right) \in W^{1} L_{\tilde{Q}}(\Omega)$, and suppose that

$$
\begin{aligned}
& \text { (ix) } b \circ u \in L_{P}(\Omega), \\
& \text { (x) } b_{k, j} \circ u_{j} \in L_{Q_{k}}^{\dagger}(\Omega), k, j=1, \ldots, m, k \neq j, \\
& \text { (xi) }\left[b_{k, k} \circ u_{k}\right] \partial_{x_{i}} u_{k} \in L_{P}(\Omega), k=1, \ldots, m, \\
& i=1, \ldots, n,
\end{aligned}
$$

where the product is to be interpreted as zero whenever ${ }_{x_{i}} u_{k}=0$.

Then $v=g \circ u$ belongs to $W^{\top} L_{P}(\Omega)$.

Proof. We first observe that, using Theorem 3.1, we can obtain the following version of Lemma 1.4 in [8]:

(i) Let $P$ be an $N$-function, and let $\Omega$ be a bounded domain in $R_{n}$ having the cone property. Then a function $f: \Omega \rightarrow R$ belongs to $W^{l} L_{P}(\Omega)$ if and only if

(a) $f \in A^{\prime}(\Omega)$,

(b) $\partial_{x_{i}}^{\prime} f \in L_{P}(\Omega), i=1, \ldots, n$.

Moreover, if $f \in W^{\mathcal{I}} L_{P}(\Omega), \partial_{x_{i}}^{\prime} f=\partial_{x_{i}} f$ almost everywhere in $\Omega$, $i=1, \ldots, n$.

Using the above instead of Lemma 1.4 in [8], the proof of Corollary 1.3 in [8] yields

(ii) Let $g: R \rightarrow R$ be a locally absolutely continuous function and let $\Omega$ be a bounded domain in $R_{n}$ having the cone property. Suppose $u \in W_{1,1}(\Omega)$, and let $v=g \circ u$. Then $v \in W^{l} L_{P}(\Omega)$ if and only if 
(*)

$$
v_{i}=\left[g^{\prime} \circ u\right] \partial_{x_{i}} u \in L_{P}(\Omega), \quad i=1, \ldots, n
$$

the product being interpreted as zero wherever $\partial_{x_{i}} u=0$. Moreover, if

(*) holds, $v_{i}=\partial_{x_{i}}{ }^{v}$ almost everywhere in $\Omega, i=1, \ldots, n$.

If we now repeat the proof of Theorem 2.1 in [8], using Theorem 5.2 (i), Theorem 5.2 (ii), 2.1 (iv), Theorem 4.1 and Theorem 4.2 instead of Lemma 1.4, Corollary 1.3, $\|20 s\|_{p} \leq\|u\|_{q_{i}}\|w\|_{q_{i}^{\prime}}$ (for suitable $u$ and $w$ ), Lemma 1.5 , and Lemma 1.6 of [8] respectively, we obtain Theorem 5.2 above.

5.3. A PARTICULAR CASE. Suppose we choose $p>1, q_{k} \geq p$, and $q_{k}^{\prime}$ such that

$$
1 / q_{k}^{\prime}+1 / q_{k}=1 / p, \quad 1 \leq k \leq m
$$

and let

$$
\begin{aligned}
P(u) & =|u|^{p}, \\
Q_{k}(u) & =|u|^{q_{k}}, \\
Q_{k}^{\dagger}(u) & =|u|^{q_{k}^{\prime},} \\
R_{k}(u) & =\left(p / q_{k}\right)|u|^{q_{k} / p},
\end{aligned}
$$

and

$$
\tilde{R}_{k}(u)=\left(p / q_{k}^{\prime}\right)|u|^{q_{k}^{\prime} / p} .
$$

Then $P, Q_{k}, Q_{k}^{\dagger}, R_{k}$ and $\tilde{R}_{k}$ satisfy conditions (i), (ii) and (iii) in Theorem 5.2. It follows that Theorem 5.2 contains Theorem 2.1 in [8] as a special case. 


\section{References}

[1] Robert A. Adams, Sobolev spaces (Pure and Applied Mathematics, 65. Academic Press [Harcourt Brace Jovanovich], New York, San Francisco, London, 1975).

[2] Shmuel Agmon, Lectures on elliptic boundary value problems (Van Nostrand, Princeton, New Jersey; Toronto; London; 1965).

[3] Thomas K. Donaldson and Neil S. Trudinger, "Orlicz-Sobolev spaces and imbedding theorems", J. Funct. Anal. 8 (1971), 52-75.

[4] Avner Friedman, Partial differential equations (Holt, Rinehart and Winston, New York, London, Sydney, 1969).

[5] Emilio Gagliardo, "Proprietà di alcune classi di funzioni in più variabili", Ricerche Mat. 7 (1958), 102-137.

[6] David Gilbarg, Neil S. Trudinger, Elliptic partial differential equations (Grundlehren der mathematischen Wissenschaften, 224. Springer-Verlag, Berlin, Heidelberg, New York, 1977).

[7]. M.A. Krasnosel'skiY and Ya.B. RutickiY, Convex functions and Orlicz spaces (translated from the first Russian edition by Leo F. Boron. Noordhoff, Groningen, 1961).

[8] M. Marcus and V.J. Mizel, "Nemitsky operators on Sobolev spaces", Arch. Rational Mech. Anal. 51 (1973), 374-370.

[9] В.П. Михайлов [V.P. МіhaTlov], Дифференииальнве уравнения в частних npousaodнбx [Partial differential equations] (Izdat. "Nauka", Moscow, 1976).

Division of External Studies,

University of Queensland,

St Lucia,

Queensland 4067,

Australia. 